Document downloaded from:

http://hdl.handle.net/10251/172310

This paper must be cited as:

Herrera-Granda, ID.; Lorente-Leyva, LL.; Peluffo-Ordóñez, DH.; Alemany Díaz, MDM. (2021). A Forecasting Model to Predict the Demand of Roses in an Ecuadorian Small Business Under

Uncertain Scenarios. Lecture Notes in Computer Science. 12566:245-258.

https://doi.org/10.1007/978-3-030-64580-9_21

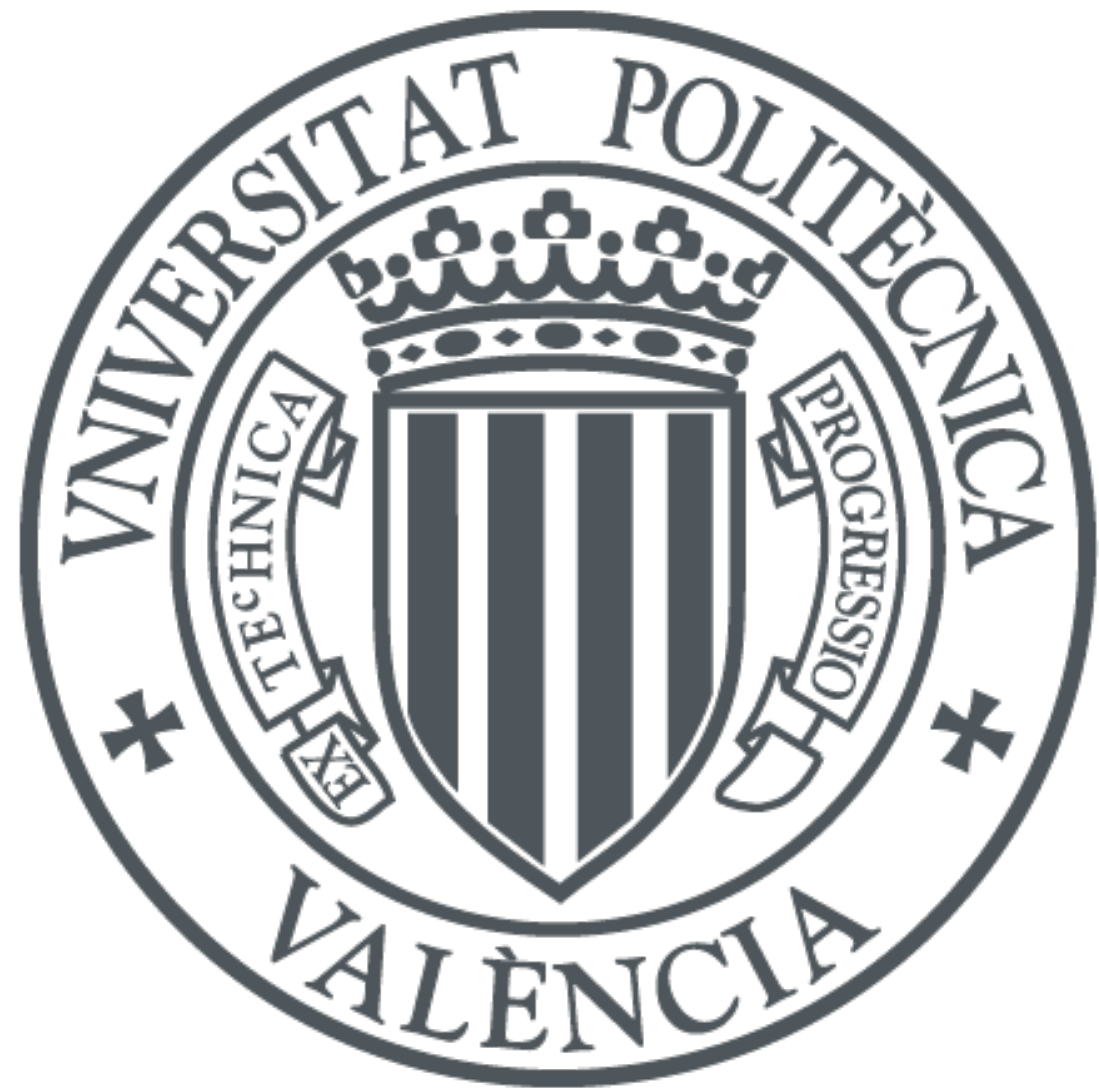

The final publication is available at

https://doi.org/10.1007/978-3-030-64580-9_21

Copyright Springer-Verlag

Additional Information 


\section{Metadata of the chapter that will be visualized in SpringerLink}

\begin{tabular}{|c|c|c|}
\hline Book Title & \multicolumn{2}{|c|}{ Machine Learning, Optimization, and Data Science } \\
\hline \multicolumn{3}{|l|}{ Series Title } \\
\hline Chapter Title & \multicolumn{2}{|c|}{$\begin{array}{l}\text { A Forecasting Model to Predict the Demand of Roses in an Ecuadorian Small Business Under Uncertain } \\
\text { Scenarios }\end{array}$} \\
\hline Copyright Year & \multicolumn{2}{|c|}{2020} \\
\hline Copyright HolderName & \multicolumn{2}{|c|}{ Springer Nature Switzerland AG } \\
\hline \multirow[t]{11}{*}{ Corresponding Author } & Family Name & Herrera-Granda \\
\hline & Particle & \\
\hline & Given Name & Israel D. \\
\hline & Prefix & \\
\hline & Suffix & \\
\hline & Role & \\
\hline & Division & \\
\hline & Organization & Universitat Politècnica de València \\
\hline & Address & Camino de Vera S/N 46022, València, Spain \\
\hline & Email & ishergra@doctor.upv.es \\
\hline & ORCID & http://orcid.org/0000-0002-4465-9419 \\
\hline \multirow[t]{10}{*}{ Author } & Family Name & Lorente-Leyva \\
\hline & Particle & \\
\hline & Given Name & Leandro $L$. \\
\hline & Prefix & \\
\hline & Suffix & \\
\hline & Role & \\
\hline & Division & \\
\hline & Organization & Universitat Politècnica de València \\
\hline & Address & Camino de Vera S/N 46022, València, Spain \\
\hline & Email & \\
\hline \multirow[t]{10}{*}{ Author } & Family Name & Peluffo-Ordóñez \\
\hline & Particle & \\
\hline & Given Name & Diego $\mathrm{H}$. \\
\hline & Prefix & \\
\hline & Suffix & \\
\hline & Role & \\
\hline & Division & \\
\hline & Organization & Yachay Tech University \\
\hline & Address & Hacienda San José, Urcuquí, Ecuador \\
\hline & Email & \\
\hline \multirow[t]{4}{*}{ Author } & Family Name & Mar Eva Alemany \\
\hline & Particle & del \\
\hline & Given Name & Maria \\
\hline & Prefix & \\
\hline
\end{tabular}


Suffix

Role

Division

Organization

Address

\section{Email}

Universitat Politècnica de València

Camino de Vera S/N 46022, València, Spain

Abstract

Ecuador is worldwide considered as one of the main natural flower producers and exporters -being roses the most salient ones. Such a fact has naturally led the emergence of small and medium sized companies devoted to the production of quality roses in the Ecuadorian highlands, which intrinsically entails resource usage optimization. One of the first steps towards optimizing the use of resources is to forecast demand, since it enables a fair perspective of the future, in such a manner that the in-advance raw materials supply can be previewed against eventualities, resources usage can be properly planned, as well as the misuse can be avoided. Within this approach, the problem of forecasting the supply of roses was solved into two phases: the first phase consists of the macro-forecast of the total amount to be exported by the Ecuadorian flower sector by the year 2020, using multi-layer neural networks. In the second phase, the monthly demand for the main rose varieties offered by the study company was micro-forecasted by testing seven models. In addition, a Bayesian network model is designed, which takes into consideration macroeconomic aspects, the level of employability in Ecuador and weather-related aspects. This Bayesian network provided satisfactory results without the need for a large amount of historical data and at a lowcomputational cost. 


\title{
A Forecasting Model to Predict the Demand of Roses in an Ecuadorian Small Business Under Uncertain Scenarios
}

\author{
Israel D. Herrera-Granda ${ }^{1(\bigotimes)}\left(\mathbb{D}\right.$, Leandro L. Lorente-Leyva ${ }^{1}$, \\ Diego H. Peluffo-Ordóñez ${ }^{2}$, and Maria del Mar Eva Alemany ${ }^{1}$ \\ ${ }^{1}$ Universitat Politècnica de València, Camino de Vera S/N 46022, València, Spain \\ ishergra@doctor.upv.es \\ 2 Yachay Tech University, Hacienda San José, Urcuquí, Ecuador
}

\begin{abstract}
Ecuador is worldwide considered as one of the main natural flower producers and exporters -being roses the most salient ones. Such a fact has naturally led the emergence of small and medium sized companies devoted to the production of quality roses in the Ecuadorian highlands, which intrinsically entails resource usage optimization. One of the first steps towards optimizing the use of resources is to forecast demand, since it enables a fair perspective of the future, in such a manner that the in-advance raw materials supply can be previewed against eventualities, resources usage can be properly planned, as well as the misuse can be avoided. Within this approach, the problem of forecasting the supply of roses was solved into two phases: the first phase consists of the macro-forecast of the total amount to be exported by the Ecuadorian flower sector by the year 2020, using multi-layer neural networks. In the second phase, the monthly demand for the main rose varieties offered by the study company was micro-forecasted by testing seven models. In addition, a Bayesian network model is designed, which takes into consideration macroeconomic aspects, the level of employability in Ecuador and weather-related aspects. This Bayesian network provided satisfactory results without the need for a large amount of historical data and at a low-computational cost.
\end{abstract}

Keywords: Bayesian-Networks-based forecasting - Demand forecast · Floriculture sector $\cdot$ Neural-Networks-based forecasting

\section{Introduction}

The floriculture sector represents one of the main income sources for the Ecuadorian agricultural sector [1]. In addition, given the geographic location and the goodness of the climate and soil, provides among its star products the high quality Ecuadorian rose, which is highly appreciated in countries like the United States, Russia, and countries of the European Union. Therefore, these figure as one of the main non-oil export products in the country, generating the creation of enterprises, employment and dynamization of the economy -especially at the Ecuador northern area. 
Nonetheless, companies dedicated to growing and processing quality roses daily face a wide variety of problems. Which in turn generate scenarios of uncertainty such as difficulty in accessing bank credit, the constant growth of inflation, the appearance of new local taxes and tariffs, the increase in the level of unemployment and the constant rotation of personnel during seasons of low demand.

Within this topic, it is also important to mention the difficulty of access to water resources, since the floriculture sector uses water in most of its processes and in the irrigation of its crops, which has often caused problems with the inhabitants and village communities who manage the irrigation canals on a community basis. Moreover, given the scarcity of access to water resources and the variety of climates in times of drought, and with the emergence of new enterprises also dedicated to this activity, the problem of access to water resources increases, and affects negatively manufacturing costs.

Despite the fact that the northern zone of Ecuador has adequate conditions in terms of soil and climate for the cultivation of high quality roses. It is important to mention that the climate is highly variable with respect to other areas of Ecuador. Specifically, two weather stations have been identified to be relatively-colder-temperature ones within the months of January to May, and the second cold season in the months of September to November, which are accompanied by increased rainfall. This variability in climate requires the use of greenhouses that seek to keep the climate of the crops stable [2, 3].

Climate variability and droughts encourage the appearance of pests, which are treated with pesticides. Within this aspect, it is also important to mention the negative impact that flower production has on the properties of the soil, since when it is overexploited it loses its chemical properties and it becomes necessary to use agricultural fertilizers, which in turn increase production costs and are a risk to human health. Therefore, all the above factors negatively affect the manufacturing costs and the quality of the final product.

In addition, we must be take into account the uncertainty of demand in the international target market comprising mainly the United States, Russia, and European Union countries. To aggravate the scenario of uncertainty in the Ecuadorian floricultural sector we can mention the variability in the international sales price, which constantly fluctuates within a constant game between offer and demand.

In an optimistic scenario, all Ecuadorian rose production would be sold and exported immediately after their production. Given the perishable nature of this product. However, unexpected events may occur such as the emergence of new rose producing countries or even pandemics or foreign trade restrictions on flower exports. It is important to note that local consumption of roses is negligible compared to exports, which may be due to the fact that international markets offer better prices for them [1].

Because of the above, there is a need to design a specific demand-forecasting model for the rose sector that considers previously mentioned variables. In addition, this model should be constantly updated to reduce uncertainty in flower production scenarios and make better demand decisions that optimize the entire Agricultural Supply Chain (ASC) and the appropriate use of water and soil for crops.

The rest of the manuscript is structured as follows: Sect. 2 shows a chronological review of the work done on the forecast of agricultural products under uncertainty scenarios. Section 3 establishes the forecasting methods used on the case study. Section 4 
shows the results obtained by applying 7 different forecasting methods and a Bayesian network (BN) specially designed for the case study. Finally, the conclusions obtained by applying the different forecasting models to the case study are discussed.

\subsection{Bibliographic Review}

Forecasting methodologies have had various applications within agricultural sciences, for example, in [4] they developed a model to manage risks in cotton production, taking into account climate uncertainty. Similarly [5] they address the problem of estimating agricultural production costs under the effects of local climatic phenomena that are highly variable such as the "El Niño" phenomenon.

The optimal allocation of land to crops has been the subject of studies, in [6] they have developed a quantitative tool for making optimal decisions. However, for its correct execution, this tool depends on an initial process to forecasting its variables, especially the variable of climate in the crops.

In [7], forecasting tools are used for market analysis and final grain prices in Germany and Poland. In the same year [8], developed a model based on Bayesian networks to represent the irrigation needs of crop farms and their effects on water sources, thus it represents a valuable tool for making future decisions on the optimal use of irrigation water. Within this same in-focus [9] they address the uncertainty of the climate variable and how its short-term forecast can improve climate control in closed and automatically controlled environments.

Subsequently, [10] used forecasting tools and market analysis techniques to assess the availability of feed for poultry and livestock in the United States. As well as analyzing the effects of the income of emerging markets who start selling feed or their raw materials in that country and its effect on costs in agricultural production. Finally, they presented their results to the U.S. Congress with possible solutions to improve the outlook. In the same year, [11] they analyze the economic impacts caused by climate change on agriculture in the state of California in the United States, in which after considering some variables on uncertain scenarios they finally give a possible short-term forecast scenario.

In [12] they discusses the effect of combining a good climate forecast, simulation of future crop yields, and the judgement of local farmers to reduce the uncertainty in the future crop reality. The need to implement a model to make the decisions was eliminated because the farmers were able to make them correctly.

With the above background, the need to develop a medium and short term forecast model that is specific to the sector of quality rose production under scenarios of uncertainty becomes apparent. In this context, the use of models based on Bayesian networks, such as the one proposed by [13] seems to be appropriate. This model also incorporates a process of training and heuristic optimization of its objective indicators. Because of the application of this model, forecasts about the future state of a variable are obtained, assuming a range of probability that the forecast is correct. This type of forecast could benefit from data provided by climate agencies that constantly monitor and forecast the climate, as is the case with the project Improving Predictions Of UK Drought (IMPETUS) [14] which forecasts droughts in the United Kingdom. 
Subsequent work also addressed the problem of forecasting the market for agricultural products, for example, [15] used hybrid methods between neural networks and diffuse systems for this purpose, while in [16] analyzed and forecasted feed and milk time series using three deterministic methods. In turn, [17] they predict the performance of pastures by means of machine learning methods, whose historical series were obtained through satellite images. On the other hand, [18] they forecast the energy demand of greenhouses by means of non-linear forecasting methods.

Within this scenario of modelling production systems in an uncertain context, [19] they analyses the impact of climate change on soybean and maize production. In [20] used Bayesian networks to forecast the yield of palm oil crops. Also [21] they use Bayesian networks to forecast relative humidity in cultivated coffee.

In 2019, we can cite the work of [22] who analyses the cereal production sector in Egypt and makes long-term forecasts of cereal production using deterministic methods. On the other hand, [23] they carry out experiments to measure the response of wheat farmers to inaccurate forecasts.

On the other hand, [24] they forecast prices and yields of cereal crops in Tanzania using stochastic and multi-variable methods. In [25] they use stochastic forecasting methods and Bayesian procedures to forecast pesticide concentrations in the crop soil in real time. Using a similar approach, [26] they model a Bayesian network that allows estimate the risks in the agricultural supply chain.

Within this context of uncertainty, it is important to cite the work of [27] who determine and evaluated the factors of uncertainty that affect the performance of an agricultural enterprise. That is, for production: agro-technical requirements, scientific results, sustainability of soil fertility; for trade: forecasting and scenario analysis and modeling; for the financial area: monitoring financial conditions and reserves to compensate for fluctuating market conditions.

As for forecasting methods, there are several classifications, but the one proposed by [28] is interesting, since it establishes two main forecasting methods: deterministic models are those in which a historical series and its future behavior can be adequately modeled through mathematical expression, without influencing these methods of unknown factors. On the other hand, there are probabilistic or stochastic models in which the future values of a time series only can be expressed as a probability of being correct.

Within this classification, we have included an additional section for hybrid prediction models, which would include those models that use both deterministic and probabilistic or stochastic models, as is the case in the field of machine learning. Note that both of the above models could be classified as quantitative. However, we could also add forecasting models that consider qualitative variables such as criteria, observation or empiricism [29].

After reviewing the literature, a lack of specific works for the floricultural sector can be noted. Therefore, within the present proposal a Bayesian (BN) network will be designed that is capable of capturing the most relevant aspects of the problems faced by the floricultural companies in Ecuador, which were described in the introduction section. In addition, the performance of the $\mathrm{BN}$ will be compared with other forecasting methods found in the literature, in which methods from the field of machine learning (ML) are also found. As described in the introduction section, most aspects that cause high uncertainty 
in the demand for roses involve stochastic processes that generate scenarios of future demand uncertainty. Therefore, the present proposed model for forecasting could be classified as a Stochastic Method. However, the proposed BN model is also tested against several deterministic and even hybrid models from the ML field such as neural networks.

This proposal provides a model to forecast the demand of the Ecuadorian floricultural sector, which has gone through a rigorous validation process and can be properly implemented in small and medium sized floricultural enterprises at a low computer cost.

Indeed, one can expect that if a good forecast is available initially, uncertainty in the future demand for products would be reduced, which would translate into producers who would be supplied in advance and in the right quantities. An adequate allocation of fields for the crop in which the soil is not overexploited and the desired rotation in the use of the crops are feasible, and working personnel could be planned, avoiding frequent dismissals and hiring in seasons of high demand, i.e., stabilization of personnel. The effect of a good demand forecast on the other components of the supply chain is also beneficial, as suppliers and their suppliers could be adequately supplied and take advantage of economies of scale. Likewise, distributors could plan the use of their facilities and could even start offering their products in foreign markets by obtaining better negotiations in terms of prices of the final product and taking advantage of foreign trade facilities in terms of the use of Incoterms, or lower cost modes and form of transportation.

Additionally, it is important to mention that although this study uses the forecasting model for Bayesian networks proposed by Scott et al. [13]. That work in its conclusions section promotes future research on a procedure to determine if certain predictors should be included in the model, and requests a research on a methodology to standardize the predictors included in the BN model.

Case Study. The study company is located in the north of Ecuador, specifically in the Pedro Moncayo village; this company consists of a small business dedicated to the production of high quality roses both natural and artificially dyed which are marketed mostly abroad. One of its main objectives is to optimize the management of the postharvest process through a forecast of future demand, thus balancing its installed supply with his demand.

Currently, the rose market has experienced a wide growth, so there is a significant unsatisfied demand that the company could aim to meet through an accurate forecast.

The external variables (predictors) to be considered in this case are included in two large groups, financial and technical variables.

The financial variables: access to financing, inflation, finance and taxes, unemployment, high market competition, the selling price of the final product, and international demand.

Technical variables: access to water resources and droughts, climate variability, storms and pests, wear and tear on soil properties

\section{Materials and Methods}

This section describes the forecasting process for the case study, which has been divided into two phases. In the first phase, or macro-forecast: we used historical data of the 
monthly demand in tons for exported roses from Ecuador to all markets since the year 1990 to 2019. With this data, we forecasted the monthly demand for the year 2020, the idea of this first forecast is to have a general idea of the market for Ecuadorian roses. Which can help the company to determine the top percentage it can enter in the national market with its products, so that it can balance its offer with his demand, at a strategic level. According to the indicator Root of Medium Square Error (RMSE), the Multilayer perceptron (MLP) Neural Network method was the one that provided the best results.

In the second phase or micro-forecast, the quantities demanded of the four main varieties of roses offered by the study company are estimated. To do this, we used order data in monthly units from January 2016 to December 2019, in order to forecast the monthly units demanded for the months of 2020.

\subsection{Phase 1: Macro-Forecast of Tons of Ecuadorian Roses to Be Exported}

In this phase, we forecasted the tons to be exported from Ecuador to the various markets of the world through for the 12 months of the year 2020 using MLP. The historical database was obtained from the Ecuadorian Rose Growers Association (EXPOFLORES) [1], thus consolidating a database of tons exported from 1990 to 2019. This macro-forecast will serve as a reference framework for knowing the global rose market in which the study company can participate.

\subsection{Phase 2: Micro-Forecast of Rose Varieties Offered}

In this phase, six forecast models were tested using the RMSE: \{HoltWinters (hw), Auto arima (Arima), Exponential smoothing with 2 different optimization criteria (ets1, ets2), Linear regression (tslm), Neural Network (nnetar), Multi Layer Perceptron (MLP) \} . Note that the first five models can be classified as deterministic, while nnetar and MLP use neural networks, therefore, they could be considered as hybrid and ML models. The results of the micro-forecast phase are discussed in the results phase.

To complete this second phase, a stochastic model of the Bayesian network (BN) was designed. It considers the historical series for the year 2019 but also considers the macroeconomic aspects and level of employability in Ecuador as well as its climaterelated aspects.

Design of a Bayesian Network (BN) for the Micro-Forecast of the Demand. In order to create a forecast model based on Bayesian networks (BN) which is more accurate for the study company also it can consider more than the historical monthly demand $D_{\text {hist }}$. In addition, it needs to consider other monthly variables that reflect the problems of the Ecuadorian rose market, then we have designed a Bayesian network model (BN) implemented through the package of $\mathrm{R}$ bsts [13], which allows us to include additional variables (predictors) to the historical series provided by the company. In this BN model, we used a Markov Chain-Monte Carlo (MCMC) sampling algorithm to simulate the posterior distribution, which smoothed the predictions over a large number of potential models using the average of the Bayesian model [13]. Below in Fig. 1, the architecture of the $\mathrm{BN}$ is shown with its predictors that affect the model output. 


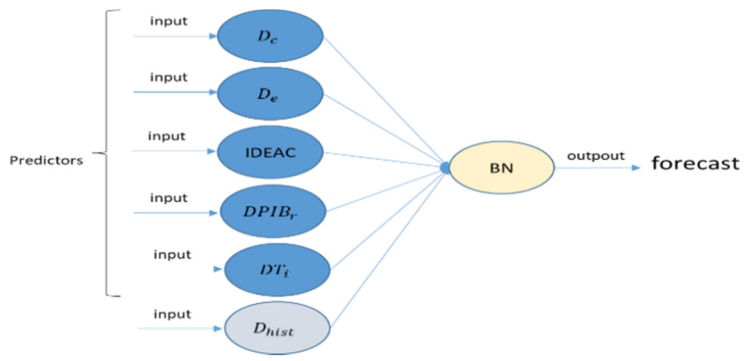

Fig. 1. BN architecture

The Climate Variability $\left(D_{c}\right)$ represents quantitatively the percentage deviation from the average in the climate of the Ecuadorian mountain range. In which there are two markedly colder seasons within the months of January to May and the second cold season in the months of September to November and consequently in those months there was an increase of rainfalls and pests, which in turn, cause the need to use greenhouses. The ideal temperature for a rose to develop and produce normally in a greenhouse is between 18 and $21{ }^{\circ} \mathrm{C}$ [30]. Therefore, in the variable $D_{c}$ the variation in temperature within the glasshouse is represented with respect to the average or ideal temperature, which should be within the aforementioned range, taking into account in this function that the minimum environmental temperature is $7{ }^{\circ} \mathrm{C}$ and the maximum is $21{ }^{\circ} \mathrm{C}$ [31]. The environmental temperature in the Ecuadorian sierra tends to be colder than inside the greenhouse, that is why in the case of this variable its decrease affects directly proportional in the model, that is to say its decrease affects negatively the crops [2, 3].

The variable Employment deviation in Ecuador $\left(D_{e}\right)$ quantitatively represents the percentage deviation from the average in the gross employment positions available in Ecuador. Therefore, its increase positively affects the result of the model [32].

The Index of Economic Activity (IDEAC) describes the country's economic reality in terms of the amount of production, so its increase has a positive effect on the model's results [33]. The IDEAC measures the percentage deviations in its values with respect to its average. Therefore, it is not necessary to normalize this variable.

Likewise, the variable Deviation of the Ecuadorian rose sector's Gross Domestic Product $\left(D P I B_{r}\right)$ measures the percentage deviation from the average contributed by the rose sector to Ecuador's gross domestic product, its increase positively affecting the model [1].

The inflation rate variable $\left(D T_{i}\right)$ reflects the increase in the prices of goods and services in Ecuador; therefore, its increase negatively affects the results of the model [33].

Process for Normalization of Predictors for the BN. The aforementioned BN were used to forecast the monthly production quantities of the different rose varieties for the year 2020. In addition, it is important to mention that the scales used in each of these variables directly affect the outcome of the model, which is why for the present case all the predictors have been normalized to keep amplitude at the same scale of a fractional number between 0 and 100 . 
However, there are also variables that indirectly affect the model proportionally, so fractional values between 0 and -100 are included. That is, if a predictor increases in magnitude it similarly affected to the estimation of the predicted quantity in the $\mathrm{BN}$, and in another case, it is said to be inversely proportional. For this reason, the inverse proportional variables affect the BN model in the opposite way and we decided to change its symbol to treat all the variables in a standard way. As shown in Fig. 2.

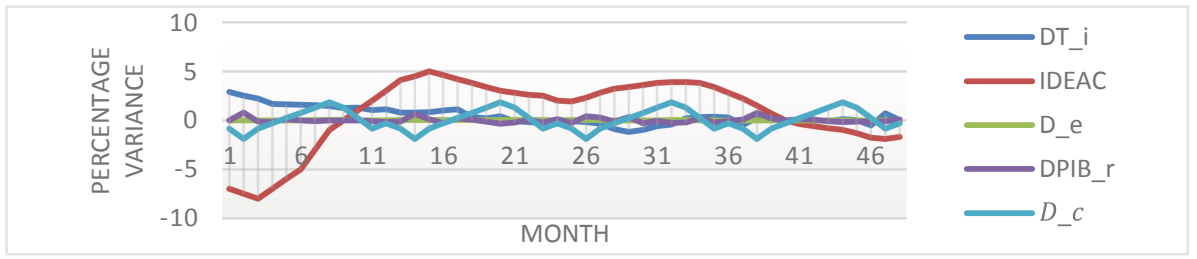

Fig. 2. BN normalized predictors

The formula used to normalize predictor values is described below. If $x$ is a variable to be used as a predictor, then $x_{i}$ will be its observations taken $m$ times corresponding to the historical series $D_{\text {hist }}$

$$
D\left(x_{i}\right)=\left[\frac{x_{i}-\bar{x}}{\bar{x}}\right] * 100, \forall i=1,2, \ldots m
$$

\section{Results}

This section shows the results of the application of the forecasting models cited in the previous section, divided into two phases: the first corresponds to the macro-forecast of the demand for the entire Ecuadorian rose market for the year 2020 in tons. While the second phase corresponds to the micro-forecast of the four varieties of roses offered by the study company.

\subsection{Results of the Macro-Forecast (Phase 1)}

From the historical data of monthly orders of roses in tons from 1990 to 2019. We want to forecast the demand for the year 2020. For this purpose, a neuronal network type MLP has been used through Rstudio's NNFOR package, since the latter has demonstrated in many similar cases its efficiency at a low computational cost, capturing trends, seasonality, and other shorter patterns within the demand by means of its Iterative Neural Filter (INF)) $[34,35]$. In this case, it was possible to reach the optimal configuration of the network using the pyramid rule and the optimal forecast result was reached in the third iteration of the re-training process.

The best result, specifically an RMSE of 1.364 was obtained with a manual parameter selection using the pyramid rule to determine the number of nodes in its three hidden 
layers: $(22,18,15)$ and 40 repetitions and an automatic selection of frequency delays. After several experiments, we noted that the retraining processes do not substantially improve the MSE of the MLP model. However, the computational cost is high for each retraining, so the following shows the result of the optimal MLP network and the forecast of rose exports for the year 2020. As shown in Fig. 3.

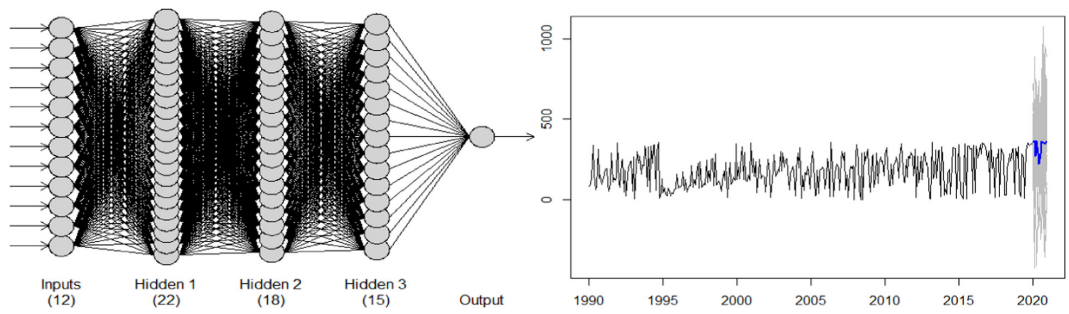

Fig. 3. Macro-forecast with MLP

\subsection{Forecast of the Demand for the Different Varieties of Roses (Phase 2)}

The above macro-forecast gave us a global picture of the rose market in 2020. Now we will work on forecasting the demand for roses for 2020 for each of the varieties of roses offered by the case study company. To do this, we used the historical monthly demands of the last three years for the four varieties of roses offered by the company, and then define a production plan to balance offer with demand in the company. The varieties of roses offered are FREEDOM, MONDIAL, PINK FLOYD and EXPLORER.

By means of the TSstudio package of the statistical language R, the NNFOR package, and the MSE indicator. It was possible to test simultaneously 7 forecast models: HoltWinters stats package (hw). Auto arima forecast package with autoregressive moving average model (Arima) [34]. Exponential smoothing state space model with error, trend and Seasonal, and 2 different optimization criteria (ets1, ets2). Neural Network time series forecast package(nnetar). Linear regression forecasting model using the tslm function (tslm). And Multi Layer Perceptron (MLP) without enhancement process, with only 1 hidden layer and without automatic delay selection [35]. A summary of the results obtained with the above-mentioned forecasting methods are shown in Fig. 4.

Forecasting Results with the BN Designed for the Case Study. A detail of the cumulative absolute errors (CAE) for each variable of each of the predictors and all of them together is shown in the left section of Fig. 5. It is possible to note that in all cases the CAE decreases by applying all the predictors simultaneously in comparison with incorporating these predictors individually. In this work, a code was developed that allows to include one by one of the predictors and finally, through the command compare.bsts [13], all the models have been graphed simultaneously. In addition, in the right section of the figure, the historical series and its forecast for the year 2020 have been included in the four types of roses offered. It can be noted that the historical demands have complex and stochastic structures. 


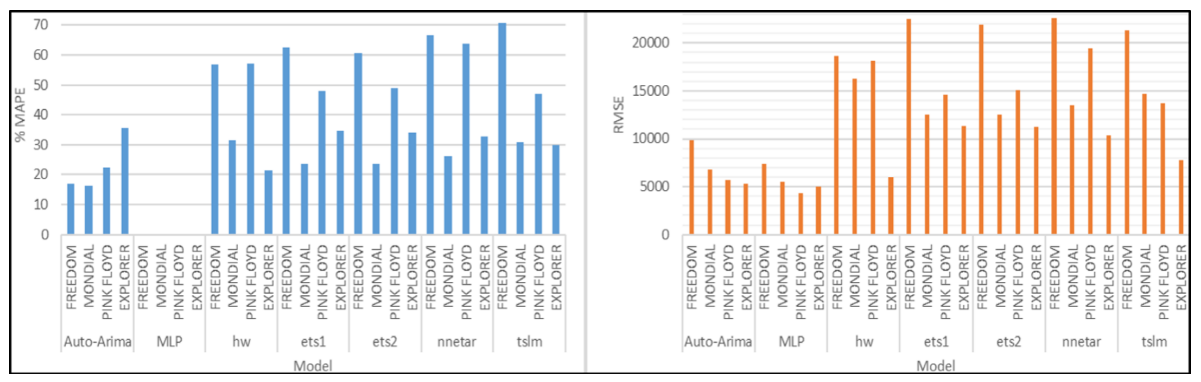

Fig. 4. Error comparison of the applied forecast models

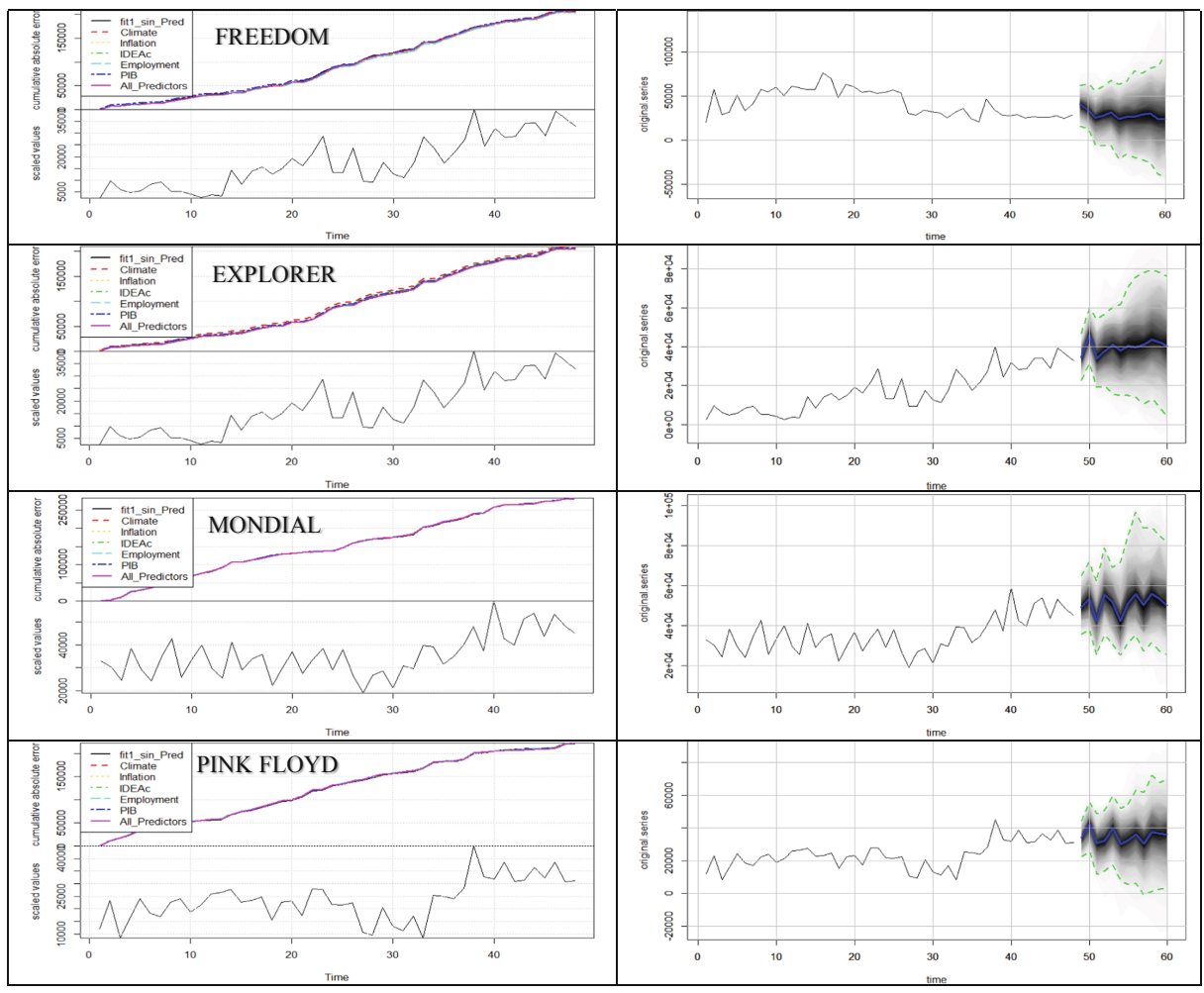

Fig. 5. Forecast of the demand with $B N$ for the varieties of roses offered.

It is important to mention that in the $\mathrm{BN}$ forecast for the four varieties of roses offered by the company. We used as a measure of the BN error, the standard deviation of the one step forward errors in the prediction (Pre-diction.sd) for the training data. Although this indicator works in a similar way to the RMSE it is not comparable with the latter since its scales differ by the amount of MCMC iterations (burn) used in the BN. As shown in Table 1. 
Table 1. Quantified errors of the proposed BN

\begin{tabular}{l|l|l}
\hline Method & Varieties & Prediction.sd \\
\hline \multirow{2}{*}{ BN } & FREEDOM & 12464,3400 \\
\cline { 2 - 3 } & MONDIAL & 7420,5040 \\
\cline { 2 - 3 } & $\begin{array}{l}\text { PINK } \\
\text { FLOYD }\end{array}$ & 5912,4030 \\
\cline { 2 - 3 } & EXPLORER & 5520,2260 \\
\hline
\end{tabular}

\section{Conclusions}

This paper proposes a model for forecasting the demand of quality Ecuadorian roses based on Bayesian networks. Which considers five exogenous variables called predictors, which include percentage deviations from climate, employment, and other macroeconomic variables such as IDEAC, inflation and gross domestic product of the rose sector. These predictors influence positively or negatively on the results of the forecast, so it is important to establish a standardization process that detects in equal conditions the variations of each predictor in the analyzed time interval, so that finally these patterns can be projected into the future. The present work is an easy implementation with low cost for the small agricultural companies and that could be constantly refined. The aspect of the incorporation of new predictors is of importance for agricultural technical professionals that could suggest new agricultural variables to incorporate and even to incorporate quantitatively their technical judgment.

The design of the mentioned $\mathrm{BN}$ with the five predictors and the historical series of monthly data of the last four years showed acceptable values in the Standard Deviation of the Errors of a step forward in the prediction (Pre-diction.sd). However, it was not possible to compare this indicator against other forecasting methods reviewed in the literature. Since due to the Bayesian nature of the $\mathrm{BN}$ its error indicators are measured within probability intervals and also because its scales differ by the amount of MCMC iterations (burn) used in the BN. Specifically in traditional forecasting models indicators such as MSE, RMSE, MAPE, among others are used. While in the BN the CAE is used measuring the error in each iteration, and on average in the BN 200 to 500 iterations are used in the experiments carried out, taking advantage of the computational performance of the MCMC algorithm. For this reason, although the RMSE indicator and the CAE used in the $\mathrm{BN}$ measure the difference between the estimation model with respect to the historical data series, in the second indicator the error is measured many more times, which is why it would be incorrect to compare the RMSE and CAE values.

For the above reason it is recommended as future work to create new error indicators in the forecast model to allow a comparison between deterministic forecasting methods and stochastic models such as BN.

In the present work, only historical data and predictors corresponding to the months from January 2016 to December 2019 are used. However, in future works, we are aimed to include in the $\mathrm{BN}$ model a new variable that reflects the global risk of pandemics that affect negatively to the production of roses. As the case, we are currently facing with the 
COVID-19 pandemic, which has not been considered in the present work and which, if it had been considered, would substantially improve the forecast for the 2020 year.

Acknowledgments. Authors of this publication acknowledge the contribution of the Project 691249, RUC-APS "Enhancing and implementing Knowledge based ICT solutions within high Risk and Uncertain Conditions for Agriculture Production Systems" (www.ruc-aps.eu), funded by the European Union under their funding scheme H2020-MSCA-RISE-2015. In addition, the authors are greatly grateful by the support given by the SDAS Research Group (www.sdas-group. com).

\section{Annexes}

A link has been included to access the historical series and the standardized predictors where each row has a monthly value. Therefore, the readers can replicate the experiment: https://utneduec-my.sharepoint.com/:f:/g/personal/idherrera_utn_edu_ec/Et2foq HulEZJpsnR8xVVxRMBZ5bGtgLvUq7dMNkmo1KyuA?e=En1Ia1.

\section{References}

1. Asociación de Productores y Exportadores de Flores: Inicio - Expoflores. https://expoflores. $\mathrm{com} /$

2. Palacios, J., Rosero, D.: Análisis de las condiciones climáticas registradas en el Ecuador continental en el año 2013 y su impacto en el sector agrícola. Estud. e Investig. meteorológicas. Ina. Inst. Nac. Meteorol. e Hidrol. Ecuador, 28, p. (2014)

3. Hidalgo-Proaño, M.: Variabilidad climática interanual sobre el Ecuador asociada a ENOS. CienciAmérica 6, 42-47 (2017)

4. Ritchie, J.W., Abawi, G.Y., Dutta, S.C., Harris, T.R., Bange, M.: Risk management strategies using seasonal climate forecasting in irrigated cotton production: a tale of stochastic dominance. Aust. J. Agric. Resour. Econ. 48, 65-93 (2004). https://doi.org/10.1111/j.1467-8489. 2004.t01-1-00230.x

5. Letson, D., Podesta, G.P., Messina, C.D., Ferreyra, R.A.: The uncertain value of perfect ENSO phase forecasts: Stochastic agricultural prices and intra-phase climatic variations. Clim. Change 69, 163-196 (2005). https://doi.org/10.1007/s10584-005-1814-9

6. Weber, E.U., Laciana, C., Bert, F., Letson, D.: Agricultural decision making in the argentine Pampas: Modeling the interaction between uncertain and complex environments and heterogeneous and complex decision makers (2008)

7. Loy, J.-P., Pieniadz, A.: Optimal grain marketing revisited a german and polish perspective. Outlook Agric. 38, 47-54 (2009). https://doi.org/10.5367/000000009787762761

8. Wang, Q.J., Robertson, D.E., Haines, C.L.: A Bayesian network approach to knowledge integration and representation of farm irrigation: 1. Model development. WATER Resour. Res. 45 (2009). https://doi.org/10.1029/2006wr005419

9. Keesman, K.J., Doeswijk, T.: uncertainty analysis of weather controlled systems (2010). https://www.scopus.com/inward/record.uri?eid=2-s2.0-79960073961\&doi=10.1007\% 2F978-3-642-03735-1_12\&partnerID=40\&md5=210525584472097e996a9f124f96fddb

10. Schnepf, R.: U.S. livestock and poultry feed use and availability: background and emerging issues. In: Feed Market Dynamics and U.S. Livestock Implications. pp. 1-36. Nova Science Publishers, Inc., CRS, United States (2012) 
11. Medellín-Azuara, J., Howitt, R.E., MacEwan, D.J., Lund, J.R.: Economic impacts of climaterelated changes to California agriculture. Clim. Change 109, 387-405 (2011). https://doi.org/ 10.1007/s10584-011-0314-3

12. McCown, R.L., Carberry, P.S., Dalgliesh, N.P., Foale, M.A., Hochman, Z.: Farmers use intuition to reinvent analytic decision support for managing seasonal climatic variability. Agric. Syst. 106, 33-45 (2012). https://doi.org/10.1016/j.agsy.2011.10.005

13. Scott, S.L., Varian, H.R.: Predicting the present with bayesian structural time series. Available SSRN 2304426 (2013)

14. Prudhomme, C., Shaffrey, L., Woollings, T., Jackson, C., Fowler, H., Anderson, B.: IMPETUS: Improving predictions of drought for user decision-making. International Conference on Drought: Research and Science-Policy Interfacing, 2015. pp. 273-278. CRC Press/Balkema, Centre for Ecology and Hydrology, Wallingford, Oxfordshire, United Kingdom (2015)

15. Wiles, P., Enke, D.: A hybrid neuro-fuzzy model to forecast the Soybean complex. International Annual Conference of the American Society for Engineering Management 2015, ASEM 2015. pp. 1-5. American Society for Engineering Management, Missouri University of Science and Technology, Engineering Management and Systems Engineering Department, United States (2015)

16. Hansen, B.G., Li, Y.: An analysis of past world market prices of feed and milk and predictions for the future. Agribusiness 33, 175-193 (2017). https://doi.org/10.1002/agr.21474

17. Johnson, M.D., Hsieh, W.W., Cannon, A.J., Davidson, A., Bedard, F.: Crop yield forecasting on the Canadian Prairies by remotely sensed vegetation indices and machine learning methods. Agric. For. Meteorol. 218, 74-84 (2016). https://doi.org/10.1016/j.agrformet.2015.11.003

18. Chen, J., Yang, J., Zhao, J., Xu, F., Shen, Z., Zhang, L.: Energy demand forecasting of the greenhouses using nonlinear models based on model optimized prediction method. Neurocomputing 174, 1087-1100 (2016). https://doi.org/10.1016/j.neucom.2015.09.105

19. Fodor, N., et al.: Integrating plant science and crop modeling: assessment of the impact of climate change on soybean and maize production. Plant Cell Physiol. 58, 1833-1847 (2017). https://doi.org/10.1093/pcp/pcx141

20. Chapman, R., et al.: Using Bayesian networks to predict future yield functions with data from commercial oil palm plantations: a proof of concept analysis. Comput. Electron. Agric. 151, 338-348 (2018). https://doi.org/10.1016/j.compag.2018.06.006

21. Lara-Estrada, L., Rasche, L., Sucar, L.E., Schneider, U.A.: Inferring Missing Climate Data for Agricultural Planning Using Bayesian Networks. LAND. 7 (2018). https://doi.org/10.3390/ land7010004

22. Abdelaal, H.S.A., Thilmany, D.: Grains production prospects and long run food security in Egypt. Sustain. 11 (2019). https://doi.org/10.3390/su11164457

23. Kusunose, Y., Ma, L., Van Sanford, D.: User responses to imperfect forecasts: findings from an experiment with Kentucky wheat farmers. Weather. Clim. Soc. 11, 791-808 (2019). https:// doi.org/10.1175/wcas-d-18-0135.1

24. Kadigi, I.L., et al.: Forecasting yields, prices and net returns for main cereal crops in Tanzania as probability distributions: a multivariate empirical (MVE) approach. Agric. Syst. 180 (2020). https://doi.org/10.1016/j.agsy.2019.102693

25. McGrath, G., Rao, P.S.C., Mellander, P.-E., Kennedy, I., Rose, M., van Zwieten, L.: Realtime forecasting of pesticide concentrations in soil. Sci. Total Environ. 663, 709-717 (2019). https://doi.org/10.1016/j.scitotenv.2019.01.401

26. Yang, B., Xie, L.: Bayesian network modelling for "direct farm" mode based agricultural supply chain risk. Ekoloji 28, 2361-2368 (2019) 
27. Zaporozhtseva, L.A., Sabetova, T. V, Yu Fedulova, I.: Assessment of the uncertainty factors in computer modelling of an agricultural company operation. International Conference on Information Technologies in Business and Industries, ITBI 2019. Institute of Physics Publishing, Voronezh State Agrarian University, Michurina Str. 30, Voronezh, 394087, Russian Federation (2019)

28. Box, G.E.P., Jenkins, G.M., Reinsel, G.C., Ljung, G.M.: Time series analysis: forecasting and control. Wiley (2015)

29. Hanke, J., Wichern, D.: Business forecast. Pearson Educación (2010)

30. Novagric: Invernaderos para Cultivo de Rosas. https://www.novagric.com/es/invernaderosrosas

31. Weather Spark: Clima promedio en Quito, Ecuador, durante todo el año Weather Spark. https://es.weatherspark.com/y/20030/Clima-promedio-en-Quito-Ecuadordurante-todo-el-año

32. Instituto Nacional de Estadísticas y Censos-INEC: Encuesta Nacional de Empleo, Desempleo y subempleo-ENEMDU. https://www.ecuadorencifras.gob.ec/empleo-diciembre-2019/

33. Central Bank of Ecuador: Central Bank of Ecuador. www.bce.fin.ec

34. Hyndman, R., Athnasopoulos, G.: Forecasting: Principles and Practice. OTexts, Australia (2018)

35. Herrera-Granda, I.D., et al.: Artificial neural networks for bottled water demand forecasting: a small business case study. In: Rojas, I., Joya, G.C.A. (eds.) International Work-Conference on Artificial Neural Networks, pp. 362-373. Springer, Canaria (2019) 


\section{Author Queries}

Chapter 21

\begin{tabular}{|c|l|c|}
\hline Query Refs. & Details Required & Author's response \\
\hline AQ1 & $\begin{array}{l}\text { This is to inform you that corresponding author has been identified } \\
\text { as per the information available in the Copyright form. }\end{array}$ & \\
\hline AQ2 & $\begin{array}{l}\text { Please check and confirm if the authors Given and Family names } \\
\text { have been correctly identified. }\end{array}$ & $\begin{array}{l}\text { Per Springer style, both city and country names must be present } \\
\text { in affiliations. Accordingly, we have inserted the country name } \\
\text { "Spain" in affiliation "1". Please check and confirm if the inserted } \\
\text { country name is correct. If not, please provide us with the correct } \\
\text { country name. }\end{array}$ \\
\hline AQ3
\end{tabular}

